\title{
Aktuelle Entwicklungen von MEMS IR-Sensorkomponenten
}

\author{
Martin Schädel' ${ }^{1}$, Julia Baldauf ${ }^{1}$, Li Long ${ }^{1}$, Jan Freitag ${ }^{1}$, Nicole Thronicke ${ }^{1}$, Neckermann Kristin ${ }^{1}$, \\ Sabine Nieland ${ }^{1}$, André Grün ${ }^{1}$, Indira Käpplinger ${ }^{1}$, Thomas Ortlepp ${ }^{1}$ \\ ${ }^{1}$ CiS Forschungsinstitut für Mikrosensorik GmbH, Konrad-Zuse-Straße 14, 99099 Erfurt, \\ Deutschland
}

\begin{abstract}
Zusammenfassung
Dieser Beitrag gibt einen kurzen Überblick zu der Thematik MEMS-basierter IR-Gassensoren und Sensorkomponenten und berichtet ausführich über den Stand aktueller Entwicklungen des CiS Forschungsinstitutes in diesem Themenfeld. Es werden verschiedene, auf thermischer Emission basierende IR-Strahler vorgestellt, die sich z.B. durch herausragenden Minaturisierungsgrad oder Wafer-Level-Packaging hervorheben. Als Alternative werden auch Ergebnisse von IR-Strahlern präsentiert, die durch Drucktechniken auf dünnen Membranträgern erzeugt werden.

Auf Detektionsseite kommen Thermopiles zum Einsatz, die ebenfalls auf Waferebene hermetisch gehaust werden können. Der Siliziumdeckel kann neben der mechanischen Schutzfunktion dabei gleichzeitig als Träger für optische Filterschichten oder siliziumintegrierte Infrarot-Optiken dienen. Im Beitrag wird ein Einblick in die eingesetzten Technologien gegeben und es werden die bisher erreichten Bauteileigenschaften vorgestellt.
\end{abstract}

Keywords: Gassensorik, optische Messverfahren, Infrarotemitter, Thermopiles, Packaging

\begin{abstract}
Motivation
Infrarotsensoren gewinnen zunehmend an Bedeutung, inbesondere in Form von Gassensoren im Bereich der Medizintechnik und Umweltsensorik [1,2]. Ermöglicht wird dies vor allem durch die zunehmende Miniaturisierung, die neben dem Energiebedarf auch die Kosten pro Baugruppe weiter reduziert. Hinzu kommt ein wachsendes Bewustsein der Gesellschaft für gesundheitliche und umweltliche Einflussfaktoren. Verschiedene am CiS Forschungsinstitut für Mikrosensorik durchgeführte Forschungsprojekte zielen darauf ab Technologien für die Fertigung von Infrarotkomponenten zu entwickeln, die diesen Ansprüchen gerecht werden.
\end{abstract}

\section{Miniaturisierte Infrarotemitter}

Infrarotemitter basierend auf einer leitfähigen strukturierten Schicht, welche auf eine Membran aufgebracht oder eingebettet ist, werden vielfach in der Literatur beschrieben [3, 4,5,6]. Diese Infrarotemitter können durch eine an Stegen aufgehängte Membranstruktur $[3,4]$ oder durch eine flächige Membran mit Öffnungen [5] oder ohne Öffnungen [6] ausgeführt werden. Zumeist wird für den
Widerstandsheizer eine Platinschicht
verwendet $[3,4,7]$. Durch die Verwendung von Platin als Heizelement kann der Infrarotemitter nicht vollständig in einem CMOS-kompatiblen Prozess gefertigt werden, wodurch die Kosten gegenüber einem vollständig in CMOSkompatiblen Dünnschichtprozessen gefertigter Infrarotemitter erhöht sind.

Die miniaturisierten Infrarotemitter des CiS Forschungsinstitutes für Mikrosensorik bestehen aus einer dielektrischen Membran in welche eine leitfähige Schicht aus einer leitfähigen Keramik eingebettet ist, welche als Widerstandsheizer dient und ein Aufheizen der Membran um mehrere hundert Grad ermöglicht. Abb. 1 zeigt beispielhaft eine thermische Simulation eines miniaturisierten Infrarotemitters mit einem derartigen Membranstapel unter Betriebsbedingungen. Die erreichbare Temperatur und der Widerstand des Bauteiles können durch eine Strukturierung der leitfähigen Schicht verändert werden.

Die Fertigung der Emitter erfolgte durch die Abscheidung der dielektrischen Schichten und der leitfähigen Schicht auf einen Siliziumwafer und der anschließenden rückseitigen Strukturierung des Siliziums mit Hilfe eines Inductive Coupled Plasmaätzverfahrens. Durch die Ausnutzung dieses Ätzverfahrens können 
nahezu beliebige Membrangeometrien realisiert werden (vgl. Abb. 2).
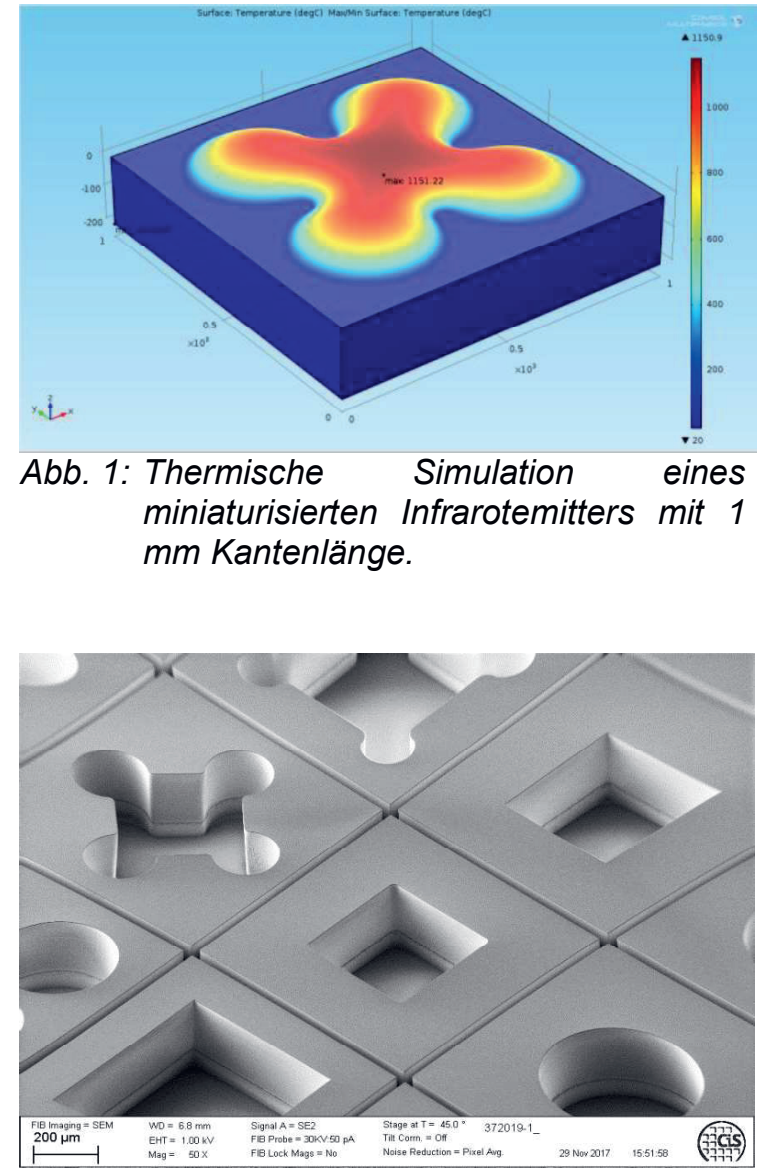

Abb. 2: Rasterelektronenmikroskopaufnahme der Rückseiten der freigestellten Membranen verschiedener Versuchsgeometrien.

\section{Druckbare Materialsysteme für Infrarotemitter}

Alternativ zu den Infrarotemittern basierend auf Dünnschichttechnologien untersucht das CiS auch Dickschichttechnologien für die Fertigung von Widerstandsheizerstrukturen für Infrarotemitter.

Bei den untersuchten Technologien handelt es sich vornehmlich um den Siebdruck von leitfähigen Pastensystemen (Silberpasten und Platinpasten). Aufgrund der CMOSInkompabilität kann der Druck hier esrt nach der Mikrostrukturierung des Siliziumträgers erfolgen.

Zunächst wird eine Passivierung in einem Dünnschichtprozess auf Siliziumwafern abgeschieden, anschließend werden Siliziummembranen mittels $\mathrm{KOH}$-Ätzen von der Rückseite erzeugt.

Diese Pasten werden mittels Siebdruck auf die Membranen appliziert und mit speziell an die Bauteile angepassten Temperaturprofilen gesintert. Eine derartige gedruckte
Widerstandsheizerstruktur bestehend aus einer Platinpaste ist in Abb. 3 gezeigt.

Unter Betrieb erhitzen die gedruckten Widerstandsheizerstrukturen die Membran auf mehrere hundert Grad (vgl. Abb. 4) und senden in einem breiten Wellenlängenbereich infrarote Strahlung aus (vgl. $A b b$. 5). Die in Abb. 5 zu erkennenden Interferenzeffekte sind von der Dicke der verwendeten Siliziummembranen abhängig.

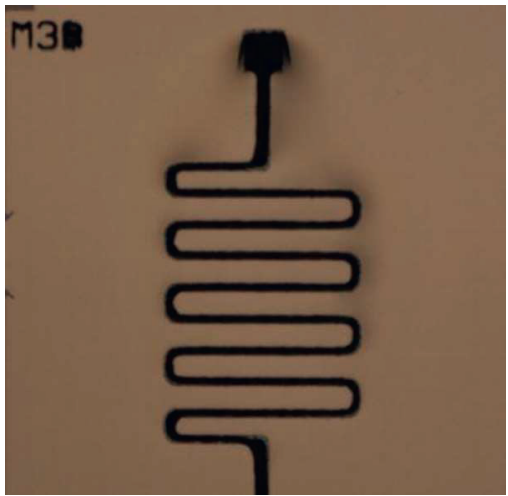

Abb. 3: Lichtmikroskopaufnahme einer siebgedruckten Platinpaste Widerstandsheizerstruktur.

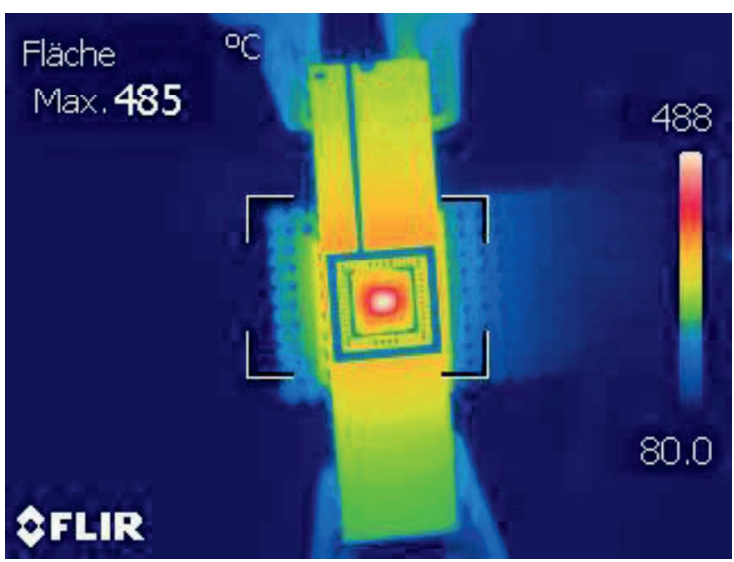

Abb. 4: Infrarotkameraaufnahme

eines gedruckten Strahlers im Betrieb.

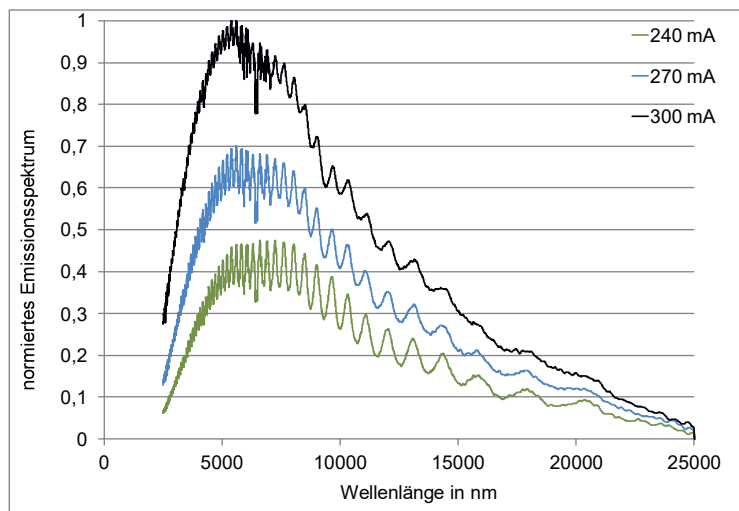

Abb. 5: Normierte Emissionsspektren eines gedruckten Strahlers mit Betrieb bei verschiedenen Stromstärken. 


\section{Thermopiles}

Thermopiles werden in vielen Anwendungen als Detektoren verwendet. Diese Anwendungen umfassen unter anderem die nicht-dispersive Infrarotgasanalyse [8,9] und die thermoelektrische Energieerzeugung [10]. Thermopilesensoren bestehen aus einer Reihenschaltung von Thermoelementen. Diese Thermoelemente wiederum werden dadurch realisiert, dass zwei thermoelektrisch verschiedene Materialien in elektrischen Kontakt gebracht werden. Ein Ende jedes Thermoelementes befindet sich auf dem Siliziumrahmen, welcher zur Kühlung dieses Endes führt, das andere Ende liegt auf einer Membran, welche zu einer thermischen Entkopplung dieses Endes führt. Bei Bestrahlung mit Licht erwärmt sich dieses Ende des Thermoelementes. Durch die Temperaturdifferenz zwischen den beiden Enden des Thermoelementes wird eine Spannung erzeugt. Die Spannung des gesamten Thermopiles ist von der Spannung der einzelnen Thermoelemente und somit von der Temperaturdifferenz, der Anzahl der Thermoelemente und der verwendeten Materialien abhängig.

Thermopiles werden am CiS Forschungsinstitut basierend auf verschiedenartig dotiertem polykristallinen Silizium erzeugt.

Die Verwendung des Materiales Silizium hat einige Vorteile, wie einen großen Seebeckkoeffizienten und vergleichsweise geringe Wärmeleitfähigkeit des polykristallinen Siliziums. Ein weiterer Vorteil ist die breite Verteilung und das gute Verständnis der Polysilizium-Dünnschichttechnologie, durch die es möglich ist große Thermowiderstände zu realisieren, in massentauglichen CMOSProzessen zu fertigen und Thermopiles kostengünstiger zu erzeugen als dies mit einer Technologie basierend auf Bismut-TelurLegierungen oder Bismut-AntimonLegierungen der Fall wäre. Darüber hinaus sind auf polykristallinem Silizium basierende Thermopiles hochtemperaturstabiler als Bismut-Antimon-basierende und Bismut-Telurbasierende Thermopiles und können ein kürzeres Ansprechverhalten erreichen.

Als Substratmaterial für die Dünnschichtabscheidungen dienen einkristalline Siliziumwafer mit einer <100> Kristallorientierung. Auf diese wird zunächst ein dielektrischer Schichtstapel aufgebracht, anschließend wird eine erste polykristalline Siliziumschicht aufgebracht, dotiert und strukturiert. Daran anschließend wird eine weitere dielektrische Schicht aufgebracht und es erfolgt eine Strukturierung, so dass ein
Kontakt zur nachfolgend abgeschiedenen dotierten polykristallinen Siliziumschicht ermöglicht wird. Diese zweite polykristalline Schicht wird mit einer weiteren dielektrischen Schicht vor Umwelteinflüssen geschützt. Die Freistellung der Membran erfolgt durch eine Kombination aus $\mathrm{KOH}$-Ätzprozess mit einem anschließenden Inductive Coupled Plasmaätzprozess.

Die wichtigsten Schritte des Prozessablaufes der Fertigung eines Thermopiles sind schematisch in Abb. 6 dargestellt und ein Beispiel für einen gefertigten Thermopile ist in Abb. 7 zu sehen. Die entstehenden Thermopiles weisen eine sehr dünne Membran einer Dicke von weniger als $2 \mu \mathrm{m}$ auf.

Um diese Membranen zu schützen kann eine Verkapselung auf Modulebene oder Waferebene erfolgen.

Darüber hinaus werden in laufenden Forschungsvorhaben durch eine verringerte Membrangröße und eine integrierte Optik (vgl. Abb. 8) Thermopilesensoren mit einer hohen Messfrequenz basierend auf CMOS kompatiblen Prozessen entwickelt.

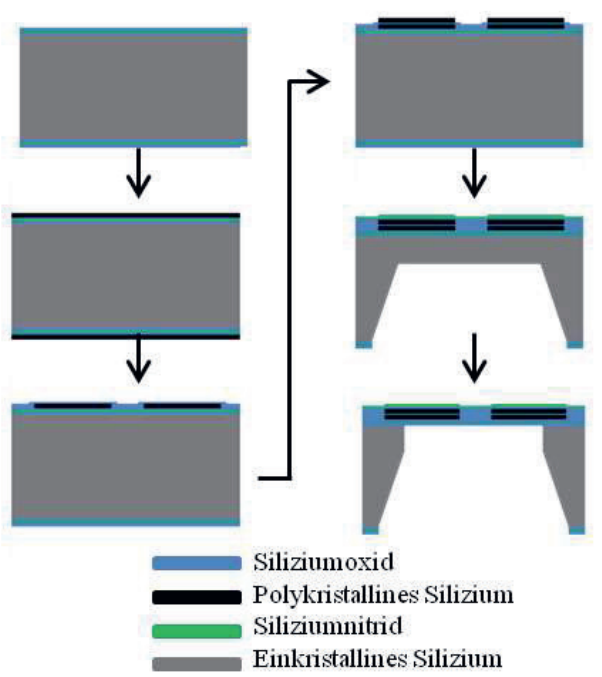

Abb. 6: Schematische Darstellung des Prozessablaufes der Fertigung eines Thermopiles. 


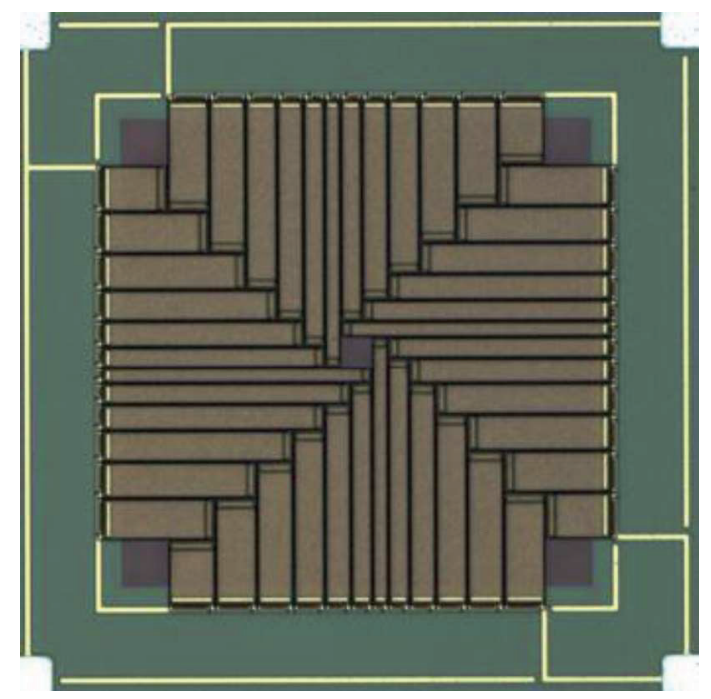

Abb. 7: Lichtmikroskopaufnahme eines Thermopiles.

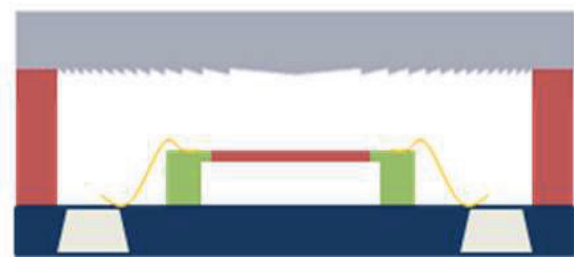

Abb. 8: Schematische Darstellung eines Thermopiles mit integrierter Optik

\section{Waferlevelpackaging von Infrarotkomponenten}

Da für einige Anwendungen eine hermetische Verkapselung der Thermopiles vorteilhaft oder zwingend notwendig ist, wurden auch Untersuchungen hinsichtlich des Packaging von Thermopilesensoren durchgeführt.

Gegenüber zu kommerziell erhältlichen Thermopiles auf Basis einer Bismut-AntimonLegierung bieten die Siliziumbasierten Thermopiles dabei den entscheidenden Vorteil auch Temperaturen von $450^{\circ} \mathrm{C}$ beim Packaging mit Hilfe von Glaslot zu ohne signifikante Änderungen überstehen.

Für die Verkapselung wurde das Glaslot sowohl auf den Substratwafer (Träger), als auch auf den Kappenwafer gedruckt, was ein Waferlevelpackaging der in Abb. 9 gezeigten Komponenten (Substratwafer, Thermopile und Kappenwafer) zu einem dichten verkapselten Thermopile ermöglichte.

Da Infrarotemitter im Allgemeinen höheren Temperaturen standhalten können als Thermopiles, kann mit der für Thermopiles entwickelten Technologie problemlos auch ein Packaging der Infrarotemitter durchgeführt werden.
Sowohl Thermopilesensoren, als auch Infrarotemitter wurden bereits erfolgreich im Waferverbund verkapselt.

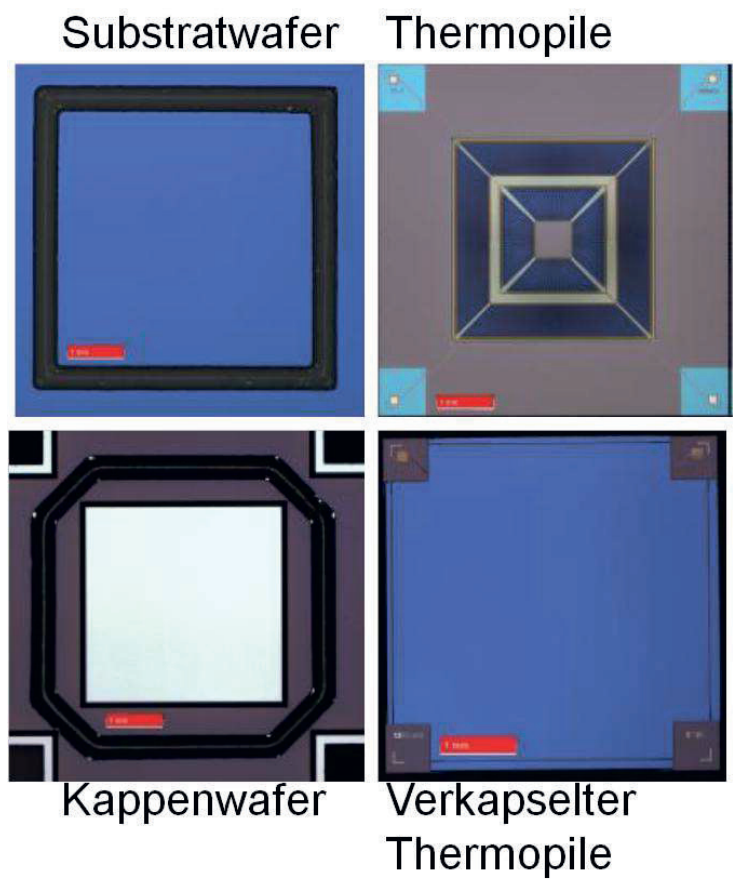

Abb. 9: Lichtmikroskopaufnahmen der für die Verkapselung notwendigen Komponenten und eines auf Waferebene gekapselten Thermopiles.

\section{Photonische Kristalle}

Um die optischen Eigenschaften eines Infrarotemitters einzustellen, können photonische Kristalle verwendet werden.

Als photonischer Kristall wird ein Festkörper mit einem periodischen Verlauf des Brechungsindexes bezeichnet. Photonische Kristalle können durch Strukturieren von bulkMaterialien oder durch die Erzeugung eines Metall-Dielektrikum-Metall-Schichtstapels realisiert werden. Die Größe, Form, Gitteranordnung der Strukturelemente und Materialparameter und Dicken der Schichten nehmen einen Einfluss auf die spektralen Eigenschaften des photonischen Kristalles.

Auch mit Hilfe von strukturiertem Silizium können photonische Kristalle erzeugt werden. $\mathrm{Am}$ CiS werden diese photonischen Kristallstrukturen zunächst als Lochstrukturen in Silizium realisiert. Dazu wurden umfangreiche Simulationen mit einem kommerziell erhältlichen Programm (VirtualLab von LightTrans International UG (www.lighttrans.com) Built 7.0.035.) durchgeführt.

Da nach dem Kirchhoffschen Gesetz im thermischen Gleichgewicht die Emission eines Materials seiner Absorption entspricht, kann für die Bestimmung der möglichen Emission einer 
Struktur eine Simulation der Absorption herangezogen werden.

Als Strukturen wurden kreisförmige Löcher verschiedener Durchmesser in quadratischen Gittern mit verschiedener Periode (Abstand Kreismitte zu Kreismitte) in verschiedenartig dotiertem Silizium mit unterschiedlichen Dicken simuliert. Außerdem wurden mit Hilfe der Simulationen Toleranzanalysen hinsichtlich des Einflusses von Strukturfehlern durch den Ätzprozess untersucht.

Abb. 10 zeigt Simulationen von $12 \mu \mathrm{m}$ dicken Siliziumschichten mit $10^{20}$ Phosphoratomen pro $\mathrm{cm}^{3}$, einer Lochstruktur (Durchmesser $10 \mu \mathrm{m})$ und verschiedener Periode.

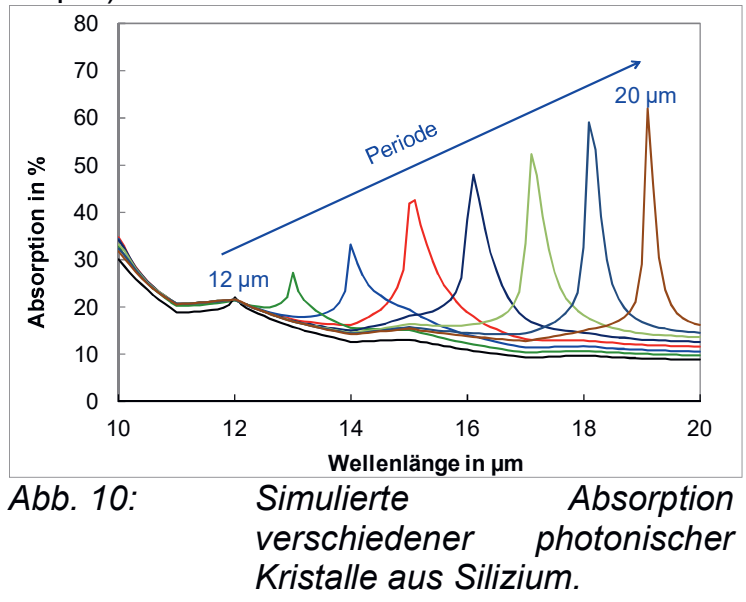

\section{Danksagung}

Die Forschungs- und Entwicklungsarbeiten für die vorgestellten Infrarotkomponenten wurden im Rahmen mehrerer Projekte gefördert

-Miniaturisierter InfraRot-Emitter-Chip (MIREC), InnoKom MF160085

-Evaluierung druckbarer Materialsysteme für IR-Emitter, InnoKom VF160028

-Schneller Infrarotsensor mit integrierter Optik, InnoKom 49MF180032

-Entwicklung einer CMOS-kompatiblen Thermopile-Infrarotsensoren Stapeltechnologie für Gasdetektoren, InnoKom MF150013

-Sipamig System-in-Package für einen miniaturisierten Gassensor bis $200^{\circ} \mathrm{C}$, ZIM ZF4000401WM5

-Photonische Kristalle für die InfraRot Gasanalyse (PhIR), InnoKom 49MF180072

gefördert durch das Bundesministerium für Wirtschaft und Energie.

\section{Literaturnachweis}

[1] IVAM-Befragung 2017 - Europas Mikrotechnikbranche stellt sich auf Wachstum ein https://www.ivam.de/research/economic_d ata/europe_s_microtechnology_industry_si gnals growth (abgerufen am 18. Jan. 2019)

[2] B. Roussel, E. Mounier; Yole Dévelopement: Gas Sensor Technology and Market, www.yole.fr, (Feb.2016)

[3] J. Hildenbrand, J. Korvink, J. Wöllenstein, C. Peter, A. Kürzinger, F. Naumann, M. Ebert, and F. Lamprecht, IEEE Sensors Journal 10, 353-362 (2010); doi: 10.1109/JSEN.2009.2034624

[4] J. Hildenbrand, C. Peter, F. Lamprecht, A. Kürzinger, F. Naumann, M. Ebert, R. Wehrspohn, J. G. Korvink, J. Wöllenstein, Microsystem Technologies 16, 745-754 (2010); doi:

10.1007/s00542-010-1049-1

[5] P. Zhou, R. Chen, N. Wang, H. San, and X. Chen, Micromachines 7, 166-172 (2016); doi:

10.3390/mi7090166

[6] H. San, X. Chen, M. Cheng, F. Li, Proceedings of SPIE 6836, 68360N168360N-8, (2007); doi:

10.1117/12.755923

[7] J. Spannhake, O. Schulz, A. Helwig, A. Krenkow, G. Müller, and

T. Doll, Sensors 6, 405-419 (2006); doi: 10.3390/s6040405

[8] S. Udina, M. Carmona, G. Carles, J. Santander, L. Fonseca, S. Marco, Sensors and Actuators B: Chemical 134, 551-558 (2008); doi:

10.1016/j.snb.2008.05.043

[9] A. Roncaglia, F. Mancarella, G.C. Cardinali, Sensors and Actuators B: Chemical 125, 214-223, (2007); doi: 10.1016/j.snb.2007.02.018

[10] V. Leonov, T. Torfs, P. Fiorini, and C. Van Hoof, IEEE Sensors Journal 7, 650-657 (2007); doi:

10.1109/JSEN.2007.894917 